\title{
A Gamificação como Estratégia para a Educação em Segurança e Saúde no Trabalho em ECITs na Paraíbai
}

\author{
Fábio Morais Borges1, Lebiam Tamar Gomes Silva2, Milena Trigueiro \\ Tavares3, Juliana Moreira de Oliveira3, João Pedro dos Santos Cipriano4
}

1Departamento de Engenharia de Produção, Universidade Federal da Paraíba (UFPB) CEP: 58051-900. Cidade Universitária - João Pessoa - PB - Brasil.

2Departamento de Habilitação Pedagógica, Universidade Federal da Paraíba (UFPB)

\author{
3Centro de Tecnologia, Universidade Federal da Paraíba (UFPB) \\ 4Centro de Educação, Universidade Federal da Paraíba (UFPB) \\ fabiomoraisbect.ufpb.br; lebiamsilva@ce.ufpb.br; \\ milenatrigueiro@hotmail.com; juliana_moreira@hotmail.com; \\ joao65644@gmail. $\overline{\mathrm{com}}$
}

\begin{abstract}
This essay is an account of experience of a university extension project, which had the goal of producing and disseminating knowledge on Work Safety, in an innovative manner, for $1_{\text {st }}$ and 2 nd year high school students from technical schools in Paraiba. Gamification, the application of game elements in non-gaming environments, was the chosen methodological strategy to elaborate the two proposed Gamified activities: Quizz and Treasure Hunt. The didactic resources employed in the contents learning process were an electronic magazine (Issuu), Kahoot virtual platform, QR Codes, treasure maps and question and answer cards. After the application of the project's Gamified activities, the involvement and learning in relation to the matter discussed was verified on the students.
\end{abstract}

Resumo. Este trabalho apresenta o relato de experiência de um projeto de extensão universitária, cujo objetivo geral foi o de produzir e disseminar conhecimentos sobre Segurança no Trabalho, de forma inovadora, com estudantes da $1^{a}$. e da $2 a$ séries do ensino médio de escolas técnicas da Paraíba. A gamificação, prática de aplicar elementos de jogos em ambientes que não são de jogos, foi a estratégia metodológica escolhida para a elaboração das duas atividades gamificadas propostas: Quizz e Caça ao tesouro. Os recursos didáticos empregados para a aprendizagem dos conteúdos foram uma revista eletrônica (Issuu), plataforma virtual Kahoot, QR Codes, mapas do tesouro e cartões de perguntas e respostas. Depois de aplicar as atividades gamificadas do projeto, verificou-se que houve o envolvimento e a aprendizagem dos estudantes em relação ao tema abordado.

\section{Introdução}


Os fatídicos dados de acidentes e doenças do trabalho que continuam a perturbar, mais do que a economia do país, famílias inteiras, são assustadores e grotescos. São indicadores similares a regiões que passam por conflitos e/ou guerras. Em 2015, segundo o Anuário Estatístico da Previdência Social [Anuário 2016], 612.632 acidentes de trabalho foram registrados. Já o Anuário Estatístico de Acidentes do Trabalho (Anuário 2017), criado pelo Ministério do Trabalho, em 2017 - última publicação revelou que foram 549.405 acidentes no Brasil.

Os últimos dados emitidos pelo IBGE (2017) sobre ocupação indicaram que, em 2010, no Brasil, havia 3.406.514 pessoas com idades entre 10 e 17 anos ocupadas em alguma forma de trabalho; na Paraíba, 69.508; e em João Pessoa, 6.234. A taxa de participação dos jovens de 15 a 24 anos no mercado de trabalho subiu de 55,3\%, em 1980, para 56,5\%, em 1991, e atingiu 61,1\% em 2000, seu patamar máximo na história urbana e industrial brasileira [Santos e Gimenez 2015].

Essa conjuntura, que ainda traz outros fatores, tem dificultado a redução do número de acidentes de trabalho, devido à ganância de grande parte dos empresários, que visam ao lucro acima de qualquer responsabilidade social; à pouca fiscalização das obrigações trabalhistas por parte do Ministério do Trabalho; e à queda na formação dos trabalhadores, tanto a básica como a profissionalizante. Esses e outros fatores contribuem para aumentar o número de acidentes com menores de idade ou jovens trabalhadores. Nessas condições, a maioria da grande população infantojuvenil é empurrada para atividades como assalariadas nas ocupações associadas ao trabalho manual, às extensas jornadas, aos reduzidos rendimentos e aos elevados índices de acidente de trabalho, especialmente na construção, na indústria têxtil, nas atividades agrícolas e extrativas e no comércio informal [Santos e Gimenez 2015].

Miranda et al (2012) constataram, em um estudo sobre acidentes fatais no Brasil, que a maioria dos acidentes atingiu homens jovens e produtivos, participantes ativos da força de trabalho e em atividades de maior grau de risco. Outro agravante na situação dos acidentes de trabalho no Brasil é sua ocorrência em crianças e adolescentes, conforme demonstraram Santos et al (2009). Dados da Previdência Social corroboram essa lógica, ao apresentar que, em 2015, 17.153 trabalhadores de até 19 anos se acidentaram. $\mathrm{Na}$ faixa de 20 a 24 anos, foram 83.065 acidentados, e de 25 a 29, 99.617 [Anuário 2016]. Para reforçar essa crise trabalhista, dados da Pesquisa Nacional de Saúde [Maia et al 2013], realizada em parceria entre o IBGE e o Ministério do Trabalho, em 2013, apontam que 1.676 .000 pessoas com idades entre 18 e 29 anos referiram ter sofrido acidente de trabalho entre os meses de setembro de 2012 e setembro de 2013. Um número 6,74 vezes maior do que o apresentado pela Previdência no mesmo período. Na Paraíba, foram 79.000, um número 15,75 vezes maior do que o apresentado pela Previdência.

Este trabalho relata a experiência desenvolvida no âmbito do projeto de extensão universitária Educação em segurança e saúde no trabalho: construindo o conhecimento nos futuros trabalhadores, cujo objetivo foi o de produzir e disseminar conhecimentos sobre Segurança e Saúde no Trabalho, de forma inovadora, com estudantes de duas Escolas Cidadãs Integrais Técnicas (ECITs) da Paraíba, nas cidades de João Pessoa e Bayeux. A equipe do projeto foi composta de docentes e discentes da Universidade Federal da Paraíba (UFPB), para o desenvolvimento de uma ação educativa interdisciplinar, baseada no uso de metodologias inovadoras e tecnologias digitais no ensino. O projeto atendeu a, aproximadamente, 320 estudantes da $1^{\mathrm{a}}$. e da $2^{\mathrm{a}}$. Séries do ensino médio das ECITs selecionadas. 
O texto a seguir apresenta a relevância de ações educativas, voltadas para a formação teórica e para o desenvolvimento de boas práticas em segurança e saúde no trabalho, que colaborem para preservar a saúde e a vida de adolescentes e jovens trabalhadores. Explica o conceito e as razões da escolha das estratégias metodológicas da gamificação e da aprendizagem móvel para a modelagem de duas atividades gamificadas para o ensino e a aprendizagem dos temas com os estudantes participantes do projeto. Descreve o desenvolvimento das etapas dessa ação de extensão universitária e relata cada atividade proposta, apresentando os materiais didáticos produzidos e as tecnologias digitais empregadas.

\section{Referencial teórico}

A educação, nos anos iniciais da escola, tem sido uma estratégia seguida por diversas frentes e órgãos em campanhas de conscientização sobre Segurança e Saúde no trabalho. Chen et al (2007) apontam a prevenção como a principal ferramenta para reduzir acidentes com jovens trabalhadores. Acredita-se que uma formação, mesmo que incipiente, sobre as questões e os conceitos relacionados à Segurança e à Saúde no Trabalho para as escolas de Educação Básica pode ampliar a eficiência do processo de conscientização.

Ressalte-se, no entanto, que a abordagem desses conteúdos com os estudantes desse nível de ensino escolar requer conhecimentos específicos da Pedagogia, que possibilitem a criação ou a aplicação de metodologias ativas de ensino e de tecnologias educacionais para a realização de práticas educativas inovadoras. Em resposta a esse contexto educativo, o projeto fundamentou suas bases pedagógicas nas metodologias ativas de ensino e aprendizagem. Segundo Diesel, Baldez e Martins (2017), embora em destaque nos últimos anos, as abordagens ativas de ensino e aprendizagem vêm sendo pensadas e aplicadas na educação brasileira desde os anos de 1930, com o movimento da Escola Nova.

Como alternativa para os modelos educativos tradicionais, o método ativo de ensino reúne teorias e práticas que deslocam o centro da ação educativa para o aprendiz e para a aprendizagem. Para fazer isso, o método ativo se funda em princípios como: problematização da realidade, reflexão, autonomia, mediação e trabalho em equipe, potencializando o protagonismo dos estudantes na construção do conhecimento e na interpretação e intervenção na realidade [Diesel, Baldez e Martins 2017].

Assim, elaborar estratégias didáticas inovadoras que possibilitem o engajamento e a motivação dos estudantes, durante o processo de ensino e aprendizagem e de construção do conhecimento, é um desafio educacional contemporâneo para qualquer educador. Nosso projeto tentou responder a esse desafio, a partir da concepção e da aplicação dos procedimentos metodológicos da gamificação. A escolha por essa metodologia ativa partiu da premissa de que ela tem um grande potencial para motivar os a se engajarem na era digital [Cotta Orlandi et al 2018]. No âmbito da educação, a gamificação é entendida como uma metodologia ativa de ensino e aprendizagem que utiliza elementos do design de games para criar um ambiente educativo mais rico em interatividade e possibilidades de escolha e de ação pelos estudantes, que colabore para uma aprendizagem significativa e prazerosa. [Silva et al 2015, Cotta Orlandi et al 2018, Silva, Sales e Castro 2019]. 
Em estudo sobre a aplicação de atividades gamificadas, Silva, Sales e Castro (2019) notaram que houve uma melhora significativa na aprendizagem dos conteúdos de Física. Esses autores verificaram isso por meio de um pré-teste aplicado para avaliar os estudantes antes e depois da realização de uma atividade gamificada. Rocha et al (2016) relatam que atividades gamificadas também têm sido aplicadas para o ensino de línguas estrangeiras e de Libras, como os aplicativos Duolingo e Serious LIBRAS, para treinamento médico e até para a reabilitação de pacientes.

Embora se possa criar uma atividade gamificada sem o uso de tecnologias digitais, o projeto integrou os princípios da gamificação aos da aprendizagem móvel, tomando o smartphone e a internet como principais tecnologias digitais para realizar as atividades propostas. Entendemos que o conceito de aprendizagem móvel tem a mobilidade como seu principal elemento, e não, o uso de dispositivos móveis, porque é a mobilidade que possibilita que as pessoas aprendam enquanto estão em movimento/deslocamento, rompendo com os limites físicos e fixos do espaço e do tempo para aprender [Valentim 2009]. De acordo com esse autor, essa concepção destaca melhor o que há de novo na aprendizagem móvel: a mobilidade e a conectividade, para que se possa aprender em qualquer lugar e a qualquer momento.

Segundo a Unesco (2014), a aprendizagem móvel vai integrar a educação nos próximos 15 anos, à medida que formos superando as limitações técnicas dos dispositivos móveis atuais, que comprometem seu uso como uma tecnologia educacional, tais como: autonomia das baterias, tamanho e qualidade de resolução, brilho das telas, capacidade de memória, resistência ao calor e à umidade, entre outras.

A estratégia BYOD (Bring Your Own Device) de aprendizagem móvel foi empregada para tornar possível a realização das atividades gamificadas do projeto. Com o uso dos smartphones dos próprios estudantes, incluímos a movimentação e a exploração dos espaços físicos das escolas nas atividades gamificadas propostas, que resultou em uma aprendizagem que envolveu a integração do corpo e da mente na construção do conhecimento, a partir do acesso e da análise de informações disponibilizadas em formato multimodal (textos, imagens e vídeos). A estratégia BYOD usa os dispositivos móveis dos próprios estudantes e permite que essas tecnologias digitais, que estão diariamente presentes nas salas de aula, transformem-se em tecnologias educacionais e sejam usadas em favor da aprendizagem. Trata-se de uma estratégia inovadora para equacionar dois problemas atuais da educação: a estrutura tecnológica precária ou inexistente das escolas brasileiras e o controle do uso indevido dos smartphones em sala de aula. Nessa perspectiva, o projeto buscou aplicar nas atividades propostas o conhecimento e a reflexão atual sobre inovação em educação, metodologias ativas de ensino, tecnologias digitais e aprendizagem móvel para observar as contribuições que elas podem oferecer aos processos de ensino e aprendizagem escolares.

\section{Metodologia}

O protagonismo estudantil, a aplicação e o compartilhamento do conhecimento acadêmico científico em contextos reais e a relação dialética entre teoria e prática são os princípios orientadores da extensão universitária. Essa é uma dimensão essencial da formação de qualquer profissional no Ensino Superior que, quando mobiliza a pesquisa e a produção de conhecimentos, contribui para cumprir a função social da universidade.

Para gerenciar o trabalho da equipe de estudantes desse projeto de extensão, adotou-se uma metodologia que seguiu os mesmos pressupostos da extensão 
universitária e das metodologias ativas de ensino destacadas anteriormente. Todo o planejamento das atividades desenvolvidas resultou de um processo colaborativo de trabalho, com a participação ativa dos docentes e dos discentes na definição de interesses, temas, estratégias didáticas e atividades de ensino e aprendizagem, por meio de reuniões semanais de estudo, planejamento, orientação e avaliação.

A dimensão de pesquisa no escopo do projeto consistiu na busca sistemática e na análise de ementas das disciplinas de Segurança do Trabalho das cinco Universidades mais bem conceituadas na área de Engenharia de Produção do Brasil, além de duas especializações em Engenharia de Segurança do Trabalho. Os conteúdos curriculares mais recorrentes foram selecionados como base teórica para a construção de um livro digital. Selecionamos nove conteúdos mais frequentes, a saber: Acidentes de Trabalho; Riscos; Legislação; Doenças Ocupacionais; Controle e Prevenção; Equipamentos de Proteção Coletiva (EPC); Equipamentos de Proteção Individual (EPI); Prevenção e Combate a Incêndios e Primeiros Socorros.

O livro digital foi construído utilizando-se os recursos de duas plataformas virtuais: Canva e Issuu. No Canva, foram diagramados os conteúdos abordados nas atividades gamificadas propostas pelo projeto. O layout do livro foi definido, tomando como premissa básica a ideia de priorizar o uso de elementos ilustrativos em detrimento de textos longos e com linguagem muito especializada/técnica. $O$ intuito foi de proporcionar uma leitura mais interessada pelos estudantes e uma compreensão rápida dos conteúdos abordados. Depois de feita a diagramação das páginas no Canva, elas foram inseridas no livro digital criado e publicado na plataforma Issuu. Essa plataforma possibilita a montagem simplificada de um livro digital, que pode ser compartilhado e acessado para os estudantes das ECITs lerem usando seus smartphones. Durante o desenvolvimento dessa etapa de preparação de materiais didáticos para subsidiar a aprendizagem dos conteúdos curriculares selecionados, os graduandos do projeto foram orientados para mobilizar o conhecimento produzido ao longo de seus cursos e desenvolver habilidades técnicas relacionadas com outras áreas de conhecimento.

A etapa seguinte do projeto consistiu em definir as atividades que seriam realizadas com os estudantes nas escolas selecionadas. Com base na metodologia ativa da gamificação e na reflexão sobre os diferentes tipos de atividades pedagógicas possíveis, decidimos propor um quizz - com auxílio dos recursos disponíveis na plataforma Kahoot - e uma atividade do tipo "caça ao tesouro". Essas duas atividades gamificadas elaboradas abordaram os conteúdos curriculares sobre Segurança e Saúde no Trabalho, contidos no livro digital.

Cada ECIT teve um dia reservado para realizar as duas atividades gamificadas propostas. No primeiro dia, participaram os estudantes dos Cursos Técnicos de Vendas e Cozinha. No segundo dia, os estudantes dos cursos técnicos de Mecânica e Design de móveis. A primeira atividade proposta foi a de ler o livro digital e participar de um quizz eletrônico na plataforma Kahoot. A segunda atividade gamificada foi a de "Caça ao tesouro". Para gerenciar e executar as duas atividades propostas aos estudantes, foram empregadas tecnologias digitais como smartphone, notebook, internet $\mathrm{Wi}$-Fi, dados móveis, data show, aplicativos como Google Doc e Forms, Canva, ISSUU, Kahoot, gerador e leitor de QR-Code.

A seguir, apresentamos a descrição das duas atividades gamificadas elaboradas e executadas pelos graduandos nas ECITs selecionadas pelo projeto. As aplicações da gamificação na Educação não são facilmente encontradas e compreendidas nas publicações consultadas. Por isso, este relato de experiência pretende contribuir com os 
leitores, no sentido de apresentar, de modo prático, como conseguimos traduzir nossa compreensão teórica sobre gamificação como uma metodologia ativa de ensino em uma prática educativa inovadora. Esperamos que a descrição das atividades gamificadas propostas, a demonstração dos materiais didáticos elaborados e a indicação dos recursos tecnológicos empregados auxiliem outros professores a (re)criarem essas e outras atividades gamificadas em suas salas de aula, que colaborem para o alcance de melhores resultados de aprendizagem com seus estudantes.

\section{Resultados e discussãoii}

\subsection{Atividade gamificada "Quizz"}

Quizz é um jogo de perguntas e respostas objetivas que tem o objetivo de avaliar o conhecimento sobre determinado assunto. Em geral, ele emprega níveis gradativos de dificuldade, pontuação, ranking e recompensa para incentivar o engajamento dos jogadores para o alcance de determinado resultado. O quizz eletrônico pode ser criado facilmente, em diferentes sistemas informáticos na Web, sem a exigência do domínio de conhecimentos e habilidades técnicas especializadas por parte do professor.

Para elaborar nossa atividade gamificada do quizz eletrônico, usamos os recursos das plataformas digitais Kahoot.com, Canva.com e o Issuu.com. O livro digital foi elaborado no aplicativo Canva e publicado para acesso no site do Issuu. A plataforma Kahoot possibilitou a criação de uma atividade gamificada do tipo quizz, em que utilizamos os elementos de game de níveis de dificuldade, controle do tempo para resposta, feedback imediato e ranking dos resultados. Foram elaborados quatro quizzes, que abordavam os assuntos elencados na seção anterior (metodologia).

Para realizar essa atividade, os estudantes foram divididos em equipes de cinco para responder o quizz, usando apenas um smartphone como dispositivo de conexão e registro de respostas. O quizz foi composto de dezessete perguntas - treze sobre Saúde e Segurança no Trabalho, e quatro, sobre conteúdos específicos dos cursos técnicos oferecidos pelas escolas. Cada pergunta tinha quatro alternativas de resposta. Foi necessário que as equipes de estudantes debatessem e escolhessem a resposta correta em um tempo máximo de um minuto. A própria plataforma Kahoot promove o somatório dos pontos de cada grupo e apresenta um resultado final (Figura 3). A pontuação obtida por cada equipe foi anotada em planilha eletrônica para ser contabilizada com a pontuação da segunda atividade gamificada de caça ao tesouro.

\subsection{Atividade gamificada de 'Caça ao tesouro'}

A caça ao tesouro é um jogo que envolve os jogadores na exploração de um território físico/geográfico, guiados por um mapa e por pistas que eles precisam encontrar e desvendar para conseguir chegar ao tesouro escondido. É um jogo que envolve agilidade de raciocínio e o desenvolvimento de estratégias de solução de problemas para alcançar o objetivo pretendido.

Para elaborar essa atividade gamificada, foi necessário fazer o mapeamento geográfico da escola, observando os locais mais adequados para a realização da atividade. A partir disso, com os conteúdos selecionados e as questões elaboradas, foi definido o regulamento da atividade proposta e construídos os mapas da trilha da caça ao tesouro (Figura 4), os cards com perguntas e respostas (Figura 5) e as pistas do jogo em QR-Codes. Os QR-Codes continham informações em formatos diversificados com textos, imagens e vídeos que tinham relação com os locais e com as questões a serem respondidas. 
Questões objetivas e subjetivas foram apresentadas em dez cards numerados (pergunta e resposta), que, para serem resolvidos, precisavam do auxílio de pistas (em formato de QR Code), distribuídas nos ambientes escolares que tinham relação direta com o conteúdo abordado em cada questão. Os grupos receberam o mapa do tesouro com a indicação da quantidade de pistas e de sua distribuição nos ambientes escolares. As equipes liam a pergunta do card, procuravam a dica correspondente nos ambientes escolares com o auxílio do mapa, liam o QR Code com seus smartphones, discutiam com o grupo sobre a pista e a resposta correta, registravam no card e prosseguiam para responder a pergunta do card seguinte.

Foi possível perceber que a aplicação das metodologias ativas de ensino e aprendizagem e das tecnologias digitais contribuiu para motivar os estudantes a se interessarem pelo tema proposto e, consequentemente, para a aprendizagem. As experiências vivenciadas ajudaram os graduandos do projeto de extensão universitária a compreenderem bem mais as concepções teóricas e a aplicação das metodologias ativas de ensino em contextos educativos reais. Os graduandos do Curso de Pedagogia puderam desenvolver competências e habilidades para intervir de forma mais propositiva na aplicação de tecnologias digitais em processos de ensino e aprendizagem e compreender como a gamificação pode ser inserida no exercício de suas atividades profissionais.

Com os resultados obtidos, constatamos que os estudantes das ECITs se engajaram significativamente no processo de resolução da atividade gamificada de 'caça ao tesouro'. Na atividade de leitura do livro digital, observamos que a ansiedade e a dispersão dos estudantes comprometeram o foco e a atenção necessários para potencializar a aprendizagem dos conteúdos apresentados. Por esse motivo, verificamos que optar por grupos menores de estudantes para a realização das atividades propostas é uma boa alternativa para novas edições do projeto. Também observamos que eles tiveram dificuldade de se concentrar e cansaço por parte dos estudantes, visto que estudam em horário integral, e as atividades foram aplicadas no início da tarde, depois do almoço. Entretanto, os resultados qualitativos e quantitativos, obtidos do Quizz no Kahoot e da correção dos cards respondidos na caça ao tesouro, indicaram uma aprendizagem satisfatória, mesmo diante das dificuldades apontadas.

Embora não tenha sido possível comparar a aprendizagem dos estudantes das ECITs antes e depois da realização das atividades gamificadas, outros objetivos do projeto foram devidamente alcançados. A elaboração de materiais didáticos lúdicos e que envolvessem os estudantes, tornando-os ativos em sua aprendizagem, era um ponto fundamental na metodologia construída. $\mathrm{O}$ envolvimento de estudantes de Pedagogia e de Engenharia de Produção na construção dos materiais foi um diferencial na formação desses graduandos. As atividades de extensão possibilitam levar os conhecimentos aprendidos na Universidade para outros entes da sociedade. Isso tem uma representatividade ainda maior por ser uma instituição pública. O objetivo de difundir os conhecimentos sobre Segurança e Saúde do Trabalho foi alcançado e atingiu um público com um grande potencial para trabalhar em ambientes repletos de riscos à saúde e à vida dos trabalhadores, como, por exemplo, os Cursos Técnicos de Mecânica e Design de Móveis. Propiciar a esses estudantes e futuros trabalhadores um primeiro contato com tais questões foi o grande objetivo deste projeto de extensão.

\section{Considerações Finais}


Esse projeto de extensão universitária para os graduandos da UFPB contribuiu, principalmente, para formar um profissional de Engenharia de Produção e de Pedagogia mais crítico, ativo, criativo e capaz de aplicar o conhecimento produzido na universidade para resolver problemas reais ou alcançar objetivos estabelecidos. O projeto também nos possibilitou construir um pensamento crítico e reflexivo sobre a interdisciplinaridade entre distintas áreas de conhecimento na Universidade, como a Engenharia de Produção e a Pedagogia, que se complementaram muito bem no escopo deste projeto. É válido ressaltar, ainda, que as tecnologias digitais e as metodologias ativas de ensino foram relevantes para o ensino e a aprendizagem dos temas abordados e colaboraram para a produção de atividades inovadoras.

Em relação à nossa formação acadêmica, as contribuições foram incalculáveis, porquanto possibilitaram o aprofundamento de conteúdos curriculares importantes para a formação profissional nos dois cursos de graduação envolvidos, a produção de conhecimento especializado sobre Segurança e Saúde no Trabalho e metodologias ativas de ensino apoiadas em tecnologia digitais.

\section{Referências}

Anuário Estatístico da Previdência Social (2016), Ministério da Fazenda, Secretaria de Previdência, Empresa de Tecnologia e Informações da Previdência. Brasília: MF/DATAPREV, $24^{\mathrm{a}}$ ed.

Anuário Estatístico de Acidentes do Trabalho: AEAT 2017 (2017), Ministério do Trabalho e Emprego [et al.], Brasília, $8^{\mathrm{a}}$ ed.

Chen, J; Kresnow, M; Simon, T. R; Dellinger, A. (2007), Injury prevention counselling and behavior among US children: results from the second injury control and risk survey. Pediatrics

Cotta Orlandi, Tomás Roberto; Gottschalg Duque, Cláudio; Mori Mori, Alexandre; \& de Andrade Lima Orlandi, Maria Tereza. (2018). Gamificação: uma nova abordagem multimodal para a educação. Biblios, (70), 17-30. https://dx.doi.org/10.5195/biblios.2018.447. Março.

Diesel, Aline; Santos Baldez, Alda Leila; Neumann Martins, Silvana. (2019). "Os princípios das metodologias ativas de ensino: uma abordagem teórica”. Revista

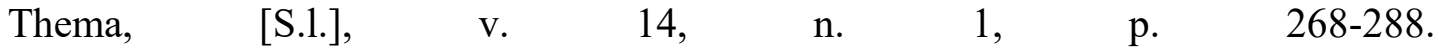
http://periodicos.ifsul.edu.br/index.php/thema/article/view/404.

IBGE - Instituto Brasileiro de Geografia e Estatística. (2017) "Censo Demográfico 2010”. ibge.gov.br . Março.

Maia, André Luís Santiago; Saito, Cezar Akiyoshi; Oliveira, Juliana Andrade; BUSSACOS, Marco Antônio; Maeno, Maria; Lorenzi, Ricardo Luiz; Santos, Sergio Antônio dos. (2013), "Acidentes de trabalho no Brasil em 2013: comparação entre dados selecionados da Pesquisa Nacional de Saúde do IBGE (PNS) e do Anuário Estatístico da Previdência Social (AEPS) do Ministério da Previdência Social". Fundacentro/Serviço de Estatística e Epidemiologia SEE. $1^{\mathrm{a}}$ ed.

Miranda, F. M. D.; Scussiato, L. A.; Kirchhof, A. L. C.; Cruz, E. D. A.; Sarquis, L. M. M. (2012) "Caracterização das vítimas e dos acidentes de trabalho fatais". Revista Gaúcha de Enfermagem. 33(2), p.45-51.

Rocha, Paul Ribeiro et al. Gamificação: um aplicativo para o ensino da Língua Brasileira de Sinais. Anais dos Workshops do Congresso Brasileiro de Informática 
na Educação, [S.1.], p. 896-900, nov. 2016. http://www.brie.org/pub/index.php/wcbie/article/view/7014. Abril.

Santos, Anselmo Luís dos, Denis Maracci Gimenez. (2015) "Inserção dos jovens no mercado de trabalho" Estudos Avançados, 29 (85).

Santos, M. E. A.; Mauro, M. Y. C; Brito, C. G.; Machado, D. C. (2009) "Trabalho precoce e acidentes ocupacionais". Escola Anna Nery Revista de Enfermagem. 13(4), p. 824-32.

Silva, F. C. P. et al. "Gamificação na Educação: a importância da teoria das emoções com estratégia de análise de jogos educacionais". In: $7^{\circ}$ Congresso Nacional de Ambientes Hipermídia para Aprendizagem, 2015, São Luís. Anais [...]. São Luís: Universidade Federal do Maranhão, 2015. p. 1-15.

Silva, João Batista da; Sales, Gilvandenys Leite; Castro, Juscileide Braga de. (2019) "Gamificação como estratégia de aprendizagem ativa no ensino de Física". Rev. Bras. Ensino Física, v. 41, n. http://www.scielo.br/scielo.php?script=sci_arttext\&pid=S1806$11172019000400502 \& \operatorname{lng}=$ en\&nrm=iso. Março.

UNESCO. Representação no Brasil. "O futuro da aprendizagem móvel: implicações para planejadores e gestores de políticas". (2014) http://unesdoc.unesco.org/images/0022/002280/228074POR.pdf. Outubro.

Valentim, H. D. "Para uma compreensão do Mobile Learning: reflexão sobre a utilidade das tecnologias móveis na aprendizagem informal e para a construção de ambientes pessoais de aprendizagem". 2009. Dissertação de Mestrado (Gestão de Sistemas de E- Learning) - Lisboa, Universidade Nova de Lisboa, 2009. https://run.unl.pt/bitstream/10362/3123/1/Hugo_Valentim_M-Learning.pdf. Outubro.

i Este projeto de extensão universitária foi financiado com recursos da Pró-Reitoria de Extensão e Assuntos Comunitários, da Universidade Federal da Paraíba, conforme edital PROBEX 2017.

ii Todos os materiais didáticos produzidos (mapas da caça ao tesouro, cards com perguntas e respostas, questões do quizz Kahoot) para as atividades propostas pelo projeto estão disponíveis para envio por email (lebiamsilva@ce.ufpb.br). 